\title{
First Measurement of Charm Production in its Fixed-Target Configuration at the LHC
}

\author{
R. Aaij et al. \\ (LHCb Collaboration)
}

(Received 19 October 2018; revised manuscript received 1 February 2019; published 2 April 2019)

\begin{abstract}
The first measurement of heavy-flavor production by the LHCb experiment in its fixed-target mode is presented. The production of $J / \psi$ and $D^{0}$ mesons is studied with beams of protons of different energies colliding with gaseous targets of helium and argon with nucleon-nucleon center-of-mass energies of $\sqrt{s_{\mathrm{NN}}}=86.6$ and $110.4 \mathrm{GeV}$, respectively. The $J / \psi$ and $D^{0}$ production cross sections in $p$ He collisions in the rapidity range $[2,4.6]$ are found to be $\sigma_{J / \psi}=652 \pm 33$ (stat) \pm 42 (syst) nb/nucleon and $\sigma_{D^{0}}=80.8 \pm 2.4$ (stat) \pm 6.3 (syst) $\mu \mathrm{b} /$ nucleon, where the first uncertainty is statistical and the second is systematic. No evidence for a substantial intrinsic charm content of the nucleon is observed in the large Bjorken- $x$ region.
\end{abstract}

DOI: $10.1103 /$ PhysRevLett.122.132002

In the high-density and high-temperature regime of quantum chromodynamics (QCD), the production of heavy quarks in nucleus-nucleus interactions is well suited to study the transition between ordinary hadronic matter and the hot and dense quark-gluon plasma (QGP). Heavy quarks are produced only in the early stages of the interaction, because their masses are significantly higher than the QGP critical temperature, $T_{c} \sim 156 \mathrm{MeV}$ [1]. Lattice QCD predictions imply that, at sufficiently high temperature, the production of heavy quark-antiquark bound states decreases due to the modification of their binding mechanism[2].

The interpretation of the charmonium $c \bar{c}$ bound states suppression, observed in nucleus-nucleus collisions at various energies[3], can be significantly sharpened by measuring charmonium yields together with the overall charm quark production[4]. The production of $D^{0}$ mesons, made of a $c$ and a $\bar{u}$ quark, reflects a large fraction of the overall charm quark production. The study of charmonium production in protonnucleus collisions on various nuclear targets, where no QGP is formed, is needed to establish the charmonium suppression patterns observed in heavy-ion collisions and to understand the mechanisms underlying charmonium production $[5,6]$.

Several effects can be studied in proton-nucleus collisions, such as the interaction of $c \bar{c}$ pairs with the target nucleons leading to a breakup of the charmonium states [7], parton shadowing (or antishadowing) in the target nucleus $[8,9]$ that modifies charmonium production, saturation effects [10], and parton energy loss [11-13].

\footnotetext{
*Full author list given at the end of the Letter.

Published by the American Physical Society under the terms of the Creative Commons Attribution 4.0 International license. Further distribution of this work must maintain attribution to the author(s) and the published article's title, journal citation, and DOI. Funded by SCOAP ${ }^{3}$.
}

In this Letter, the first measurement of heavy-flavor production in a fixed-target mode at the LHC is presented. The production of $J / \psi$ and $D^{0}$ mesons are studied in collisions of protons with energies of 4 and $6.5 \mathrm{TeV}$ incident on helium and argon nuclei at rest at center-of-mass energies of $\sqrt{s_{\mathrm{NN}}}=86.6 \mathrm{GeV}$ and $\sqrt{s_{\mathrm{NN}}}=110.4 \mathrm{GeV}$, respectively.

The $\mathrm{LHCb}$ detector $[14,15]$ is a single-arm forward spectrometer covering the pseudorapidity range $2<\eta<5$, designed for the study of particles containing $c$ or $b$ quarks. The detector elements that are particularly relevant to this analysis are the VELO surrounding the $p p$ interaction region that allows $c$ and $b$ hadrons to be identified from their characteristic flight distance, a tracking system that provides a measurement of the momentum of charged particles, two ring-imaging Cherenkov detectors that are able to discriminate between different species of charged hadrons, a calorimeter system consisting of scintillatingpad and preshower detectors, electromagnetic and hadronic calorimeters, and a muon detector composed of alternating layers of iron and multiwire proportional chambers. The system for measuring overlap with gas (SMOG) device [16] enables the injection of gases with pressure of $\mathcal{O}\left(10^{-7}\right)$ mbar in the beam pipe section crossing the silicon-strip vertex locator (VELO), allowing LHCb to operate as a fixed-target experiment. SMOG allows the injection of noble gases and therefore gives the unique opportunity to study nucleusnucleus and proton-nucleus collisions on various targets. Thanks to the boost induced by the high-energy proton beam, the LHCb acceptance covers the backward rapidity hemisphere in the center-of-mass system of the reaction from a very negative center-of-mass rapidity $y^{*} \sim-2.5$ to $y^{*} \sim 0$. Therefore, the SMOG fixed-target program offers many new opportunities of physics studies [17], including the study of heavy-quark production in the large Bjorken- $x$ region, with $x$ the fraction of the nucleon momentum carried by the target 
parton, up to $\sim 0.37$ for $D^{0}$ mesons, and the test of the intrinsic charm content of the proton $[18,19]$.

The events are triggered by the two-stage trigger system of the experiment [20]. The first level is implemented in hardware and uses information provided by the calorimeters and the muon detectors, while the second is a software trigger. The hardware trigger requires at least one identified muon for the selection of the $J / \psi \rightarrow \mu^{-} \mu^{+}$candidates, and a minimal activity in the calorimeter for the $D^{0}$ selection. The software trigger requires two well-reconstructed muons forming an invariant mass larger than $2700 \mathrm{MeV} / c^{2}$ for the $J / \psi$ selection. For the $D^{0}$ selection, it requires a wellreconstructed vertex formed by well-identified kaon and pion tracks, both of which are required to have a transverse momentum larger than $500 \mathrm{MeV} / c$ and an invariant mass between 1715 and $2015 \mathrm{MeV} / c^{2}$.

The data samples have been collected under particular beam conditions where proton bunches moving towards the detector do not cross any bunch moving in the opposite direction at the nominal $p p$ interaction point. Events with $J / \psi$ or $D^{0}$ candidates must have a reconstructed primary vertex within the fiducial region $-200 \mathrm{~mm}<z_{P V}<200 \mathrm{~mm}$, where high reconstruction efficiencies are achieved and calibration samples available. In order to suppress residual $p p$ collisions, events with activity in the backward region are vetoed, based on the number of hits in VELO stations upstream of the interaction region.

The offline selection of $J / \psi$ and $D^{0}$ candidates is similar to that used in Refs. [21,22]. Specifically, events with at least one primary vertex are selected where the primary vertex is reconstructed from at least four tracks in the VELO detector. The $J / \psi$ candidates are obtained from two oppositely signed muons forming a good-quality vertex. The well-identified muons have a transverse momentum, $p_{T}$, larger than $700 \mathrm{MeV} / c$ and are required to be consistent with originating from the primary interaction point. The kaon and pion from the $D^{0}$ decay are required to be of good quality and to come from a common displaced vertex. Tight requirements are set on the kaon and pion particle identification criteria. The $D^{0}$ candidates are selected to have a decay time larger than $0.5 \mathrm{ps}$. The measurements are performed in the range of $J / \psi$ and $D^{0}$ transverse momentum $p_{T}<8 \mathrm{GeV} / c$ and rapidity $2.0<y<4$.6.

Acceptance and reconstruction efficiencies are determined using simulated $p \mathrm{He}$ and $p \mathrm{Ar}$ events.

In the simulation, $J / \psi$ and $D^{0}$ mesons are generated using Pythia $8[23,24]$ with a specific LHCb configuration [25] and with colliding-proton beam momenta being equal to the momenta per nucleon of the beam and target in the center-of-mass frame. The decays are described by EvTGEN [26], in which final-state radiation is generated using Pнотоs [27]. The four-momenta of the $J / \psi$ and $D^{0}$ daughters are then extracted and embedded into $p$ Ar or $p \mathrm{He}$ minimum bias events that are generated with the EPOS event generator [28] using beam parameters obtained from the data. Decays of hadronic particles generated with EPOS are also described by EVTGEN. The interaction of the generated particles with the detector, and its response, are implemented using the GEANT4 toolkit $[29,30]$ as described in Ref. [31].

The $J / \psi$ detection efficiency is dependent on its polarization. Since no polarization measurement has yet been made for data collected at $\sqrt{s_{\mathrm{NN}}}$ close to $100 \mathrm{GeV}$, the polarization is assumed to be zero and no corresponding systematic uncertainty is quoted on the cross-section results. A small longitudinal polarization, described by the parameter $\lambda_{\theta}$, has been found at different energies close to $\lambda_{\theta}=-0.1$ [32-34]. Using data from Ref. [35] and assuming a value $\lambda_{\theta}=-0.1$, the measured $J / \psi$ cross section would decrease by about $1 \%$ to $2.3 \%$ depending on the $J / \psi\left(p_{T}, y\right)$ bin [36].

The prompt $J / \psi$ and $D^{0}$ signal yields are obtained from extended unbinned maximum-likelihood fits to their mass distributions. The fit functions are given by the sum of a crystal ball function [37] describing the $J / \psi$ signal, and an exponential function for the background. The $D^{0}$ signal is fitted by the sum of two Gaussian functions, and an exponential function for the background. Figure 1 shows
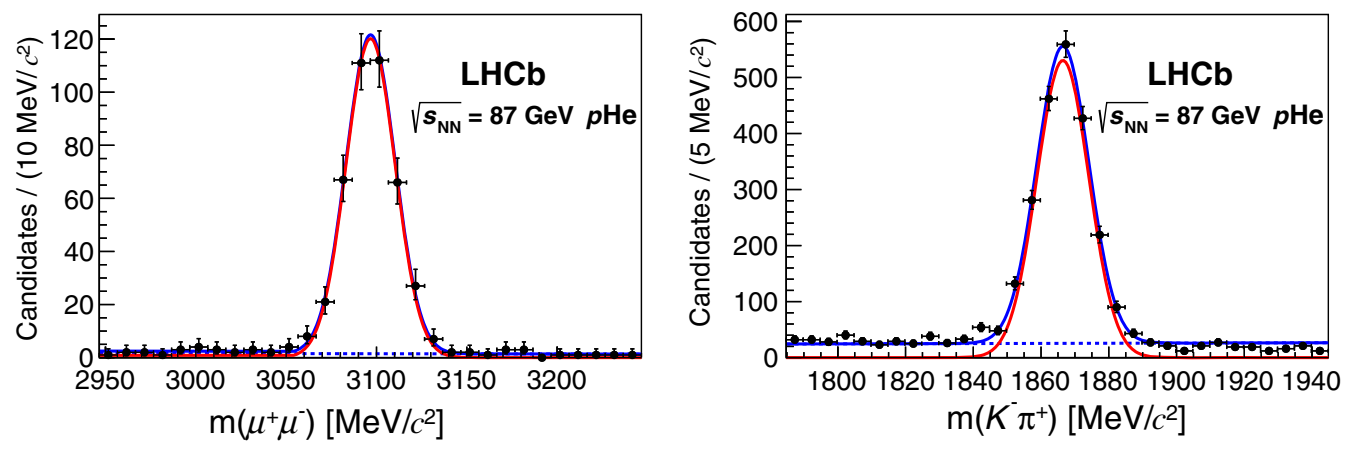

FIG. 1. Mass distributions, fitted by an extended unbinned maximum-likelihood in $\sqrt{s_{\mathrm{NN}}}=86.6 \mathrm{GeV} p \mathrm{He}$ collisions; $J / \psi \rightarrow \mu^{-} \mu^{+}$ (left); $D^{0} \rightarrow K^{-} \pi^{+}$(right). The dashed blue line corresponds to the combinatorial background, the red line to the signal, and the solid blue line to the sum of the two. 
TABLE I. Systematic and statistical uncertainties on the $J / \psi$ and $D^{0}$ yields in $\%$. Systematic uncertainties correlated between bins affect all measurements by the same relative amount. Ranges denote the minimum and the maximum values among the $y$ or $p_{T}$ bins.

\begin{tabular}{llcc}
\hline \hline Source & & $J / \psi$ & $D^{0}$ \\
\hline Correlated between bins & & & \\
Signal selection efficiency & $p \mathrm{Ar}$ & $1.4 \%$ & $1.4 \%$ \\
& $p \mathrm{He}$ & $1.1 \%$ & $1.1 \%$ \\
Tracking efficiency & $p \mathrm{Ar}$ & $1.9 \%$ & $3.5 \%$ \\
& $p \mathrm{He}$ & $1.1 \%$ & $3.2 \%$ \\
Particle identification & $p \mathrm{Ar}$ & $(1.8-1.9) \%$ & $(4.3-5.4) \%$ \\
$\quad$ efficiency & $p \mathrm{He}$ & $(0.9-1.0) \%$ & $(1.1-2.6) \%$ \\
Uncorrelated between bins & & & \\
Signal determination & $p \mathrm{Ar}$ & $(0-0.9) \%$ & $(1.6-2.6) \%$ \\
& $p \mathrm{He}$ & $(0-0.9) \%$ & $(1.6-2.5) \%$ \\
Tracking efficiency & $p \mathrm{Ar}$ & $(0.1-1.9) \%$ & $(0.2-2.6) \%$ \\
& $p \mathrm{He}$ & $(0.2-1.8) \%$ & $(0.3-2.7) \%$ \\
Simulation sample & $p \mathrm{Ar}$ & $(1.8-2.0) \%$ & $(2.4-2.5) \%$ \\
& $p \mathrm{He}$ & $(1.7-3.4) \%$ & $(2.5-2.8) \%$ \\
Particle identification & $p \mathrm{Ar}$ & $(0-1.9) \%$ & $(0-5.6) \%$ \\
$\quad$ efficiency & $p \mathrm{He}$ & $(0-0.8) \%$ & $(0-3.7) \%$ \\
Statistical uncertainties & $p \mathrm{Ar}$ & $(7.8-12.7) \%$ & $(2.8-5.8) \%$ \\
& $p \mathrm{He}$ & $(7.9-11.3) \%$ & $(4.2-10.1) \%$ \\
\hline \hline
\end{tabular}

the mass distributions obtained after all selection criteria are applied to the entire $p \mathrm{He}$ data set, with the fit functions superimposed.

The signal yields are determined in uniformly populated bins of $p_{T}$ or $y$. A coarser binning scheme is used for $J / \psi$ candidates, owing to the smaller sample size. The yields determined from the mass fit are corrected for the total efficiencies, which include the geometrical acceptance of the detector, the event trigger, the event selection, the primary vertex, the track reconstruction, and particle identification. Particle identification [38] and tracking efficiencies are obtained from control sample of $p p$ collision data. All of the other efficiencies are determined from simulation. Several sources of systematic uncertainties are considered, affecting either the determination of the signal yields or the total efficiencies. They are summarised in Table I separately for correlated and uncorrelated systematic uncertainties.

A systematic uncertainty is assigned to the signal determination. A first contribution, common to $J / \psi$ and $D^{0}$ signals, is obtained by determining the maximum contamination from residual $p p$ collisions. The systematic uncertainty related to the determination of the signal yields includes the contribution from $b$-hadron decays and the mass fit. The fraction of signal from $b$ hadrons, determined through the fit of the impact parameter distribution of the $D^{0}$ candidates with respect to the primary vertex, is $\left(0.9_{-0.9}^{+1.6}\right) \%$. The systematic uncertainty related to the mass fit is evaluated using alternative models for signal and background shapes that reproduce the mass shapes equally well.
Another source of uncertainty is associated with the accuracy of the simulation used to compute the acceptances and efficiencies. This systematic uncertainty includes the statistical uncertainty due to the finite size of the simulation sample and the differences in the distributions of the transverse momentum and rapidity between data and simulation. This systematic uncertainty is computed in each $y$ and $p_{T}$ bin. Systematic uncertainties in tracking and particle identification efficiencies are mainly related to the differences between the track multiplicity in $p \mathrm{Ar}, p \mathrm{He}$, and $p p$ collisions. The tracking systematic uncertainty also takes into account the difference in tracking efficiency between the data and the simulation.

The cross-section measurement is made for the $p \mathrm{He}$ sample only, for which the luminosity determination is available. The luminosity is determined from the yield of electrons elastically scattering off the target He atoms [39] to be $\mathcal{L}_{p \mathrm{He}}=7.58 \pm 0.47 \mathrm{nb}^{-1}$. The measured $J / \psi$ and $D^{0}$ cross sections per target nucleon within $y \in[2,4.6]$, after correction for the branching fractions $J / \psi \rightarrow \mu^{+} \mu^{-}$and $D^{0} \rightarrow K^{+} \pi^{-}$, are

$$
\begin{aligned}
\sigma_{J / \psi} & =652 \pm 33(\text { stat }) \pm 42 \text { (syst) } \mathrm{nb} / \text { nucleon } \\
\sigma_{D^{0}} & =80.8 \pm 2.4(\text { stat }) \pm 6.3 \text { (syst) } \mu \mathrm{b} / \text { nucleon } .
\end{aligned}
$$

In order to compare to previous experimental results at different energies, both $J / \psi$ and $D^{0}$ cross sections are extrapolated to the full phase-space using PYTHIA 8 with a specific LHCb tuning and with the CT09MCS PDF set [40]. The extrapolation factor is $2 f$, where $f=0.940$ for the $J / \psi$ and $f=0.965$ for the $D^{0}$, and describes the extrapolation from $y^{*} \in[-2.53,0.07]$ to the full backward (negative) rapidity hemisphere, assuming forward-backward symmetry. The full phase-space cross sections are

$$
\begin{aligned}
\sigma_{J / \psi} & =1225.6 \pm 100.7 \mathrm{nb} / \text { nucleon } \\
\sigma_{D^{0}} & =156.0 \pm 13.1 \mu \mathrm{b} / \text { nucleon }
\end{aligned}
$$

where statistical and systematic uncertainties have been added quadratically and no systematic uncertainties due to the extrapolation are included. In addition, the $D^{0}$ cross section is scaled with the global fragmentation factor $f\left(c \rightarrow D^{0}\right)=0.542 \pm 0.024$ [41], in order to obtain the $c \bar{c}$ production cross section $\sigma_{c \bar{c}}=288 \pm 24.2 \pm 6.9 \mu \mathrm{b} /$ nucleon. The last uncertainty reflects the limited knowledge of the fragmentation factor. An overview of $J / \psi$ and $c \bar{c}$ cross-section measurements at different center-of-mass energies by different experiments are shown in Fig. 2 including this measurement. The $J / \psi$ cross section is compared to a fit based on NLO NRQCD calculations [42] and the $c \bar{c}$ cross section to NLO pQCD calculations $[43,44]$. The $c \bar{c}$ cross section shows a small tension with respect to theoretical calculations as already observed at $200 \mathrm{GeV}$, while the $J / \psi$ cross-section measurement is in 

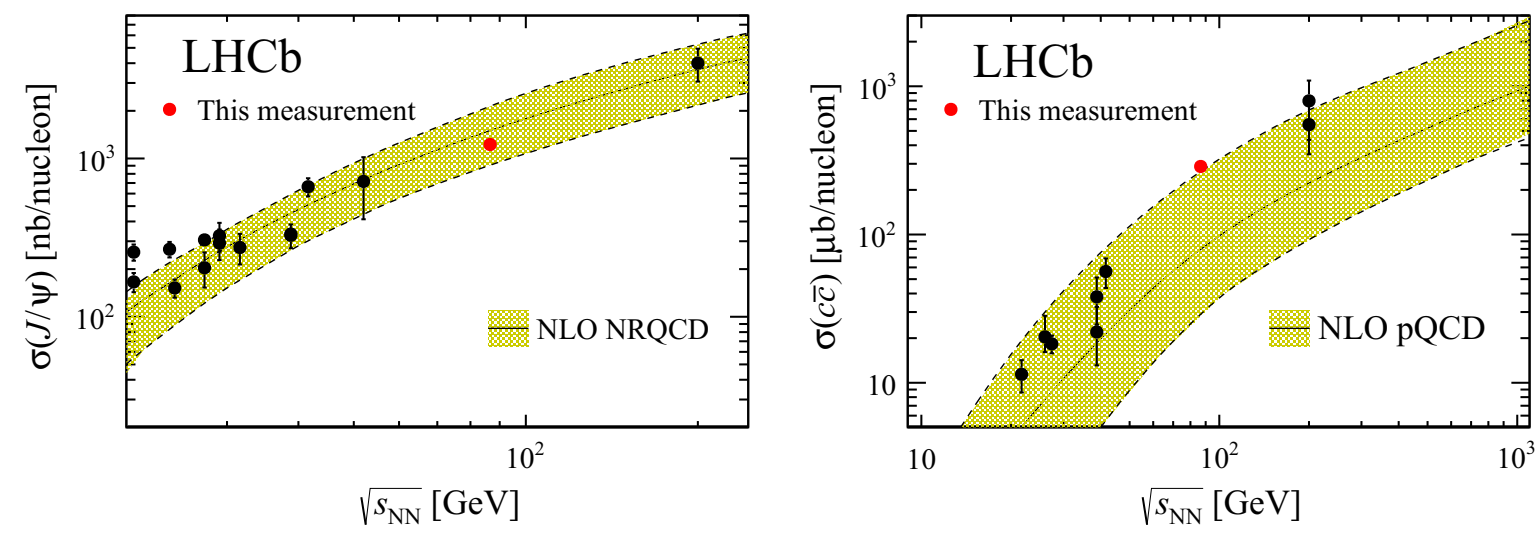

FIG. 2. Left: $J / \psi$ cross-section measurements as a function of the center-of-mass energy. Experimental data, represented by black points, are taken from Ref. [42]. The band corresponds to a fit based on NLO NRQCD calculations [42]. Right: $c \bar{c}$ cross-section measurements as a function of the center-of-mass energy. Experimental data, represented by black points, are taken from Ref. [43]. The yellow band corresponds to NLO pQCD calculations [44]. Red points correspond to the $p \mathrm{He}$ results from the present analysis.

good agreement with the fit based on NLO pQCD calculations. The $J / \psi$ differential cross sections per target nucleon obtained for the $p$ He dataset, as functions of $y^{*}$ and $p_{T}$, are shown in the two top plots of Fig. 3 and given in Ref. [36]. These results are compared with HELAC-ONIA predictions [45-47], for $p p$ (CT14NLO PDF set [48]) and $p \mathrm{He}$ (CT14NLO + nCTEQ15 PDF [49] sets) collisions. The predictions underestimate the measured total cross section. The HELAC-ONIA predictions are rescaled by a factor 1.78 in Fig. 3 to compare the shape of the distributions.
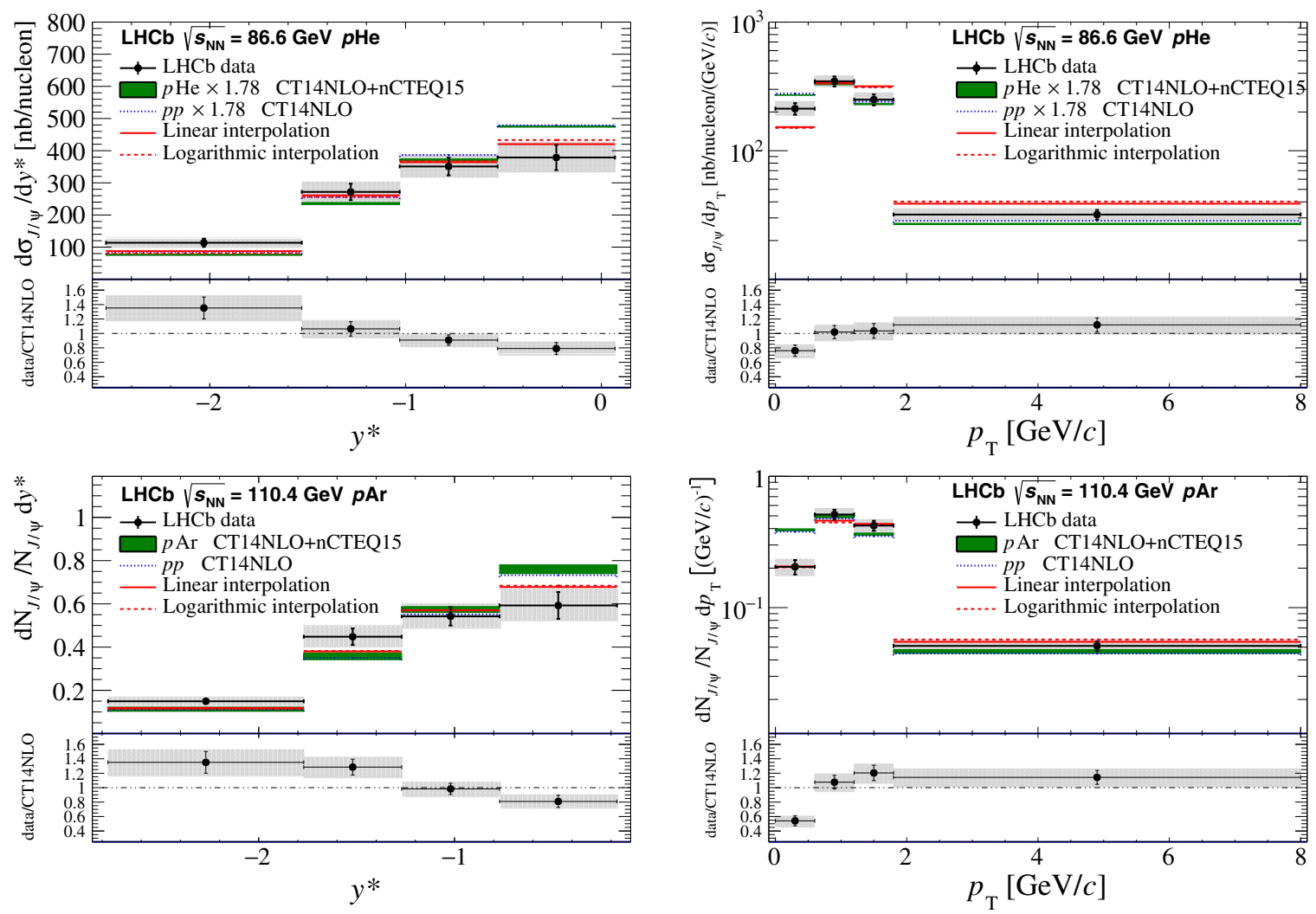

FIG. 3. Differential $J / \psi$ production cross sections for (top) $p$ He and differential $J / \psi$ yields for (bottom) $p$ Ar collisions, as a function of (left) center-of-mass rapidity $y^{*}$ and (right) transverse momentum $p_{T}$. The data points mark the bin centers. The quadratic sum of statistical and uncorrelated systematic uncertainties are indicated by the vertical black lines. The correlated systematic uncertainties are indicated by the grey area. Theoretical predictions are described in the text. The lower panel of each plot shows the ratio of data to HELAC-ONIA $p p$ predictions. 

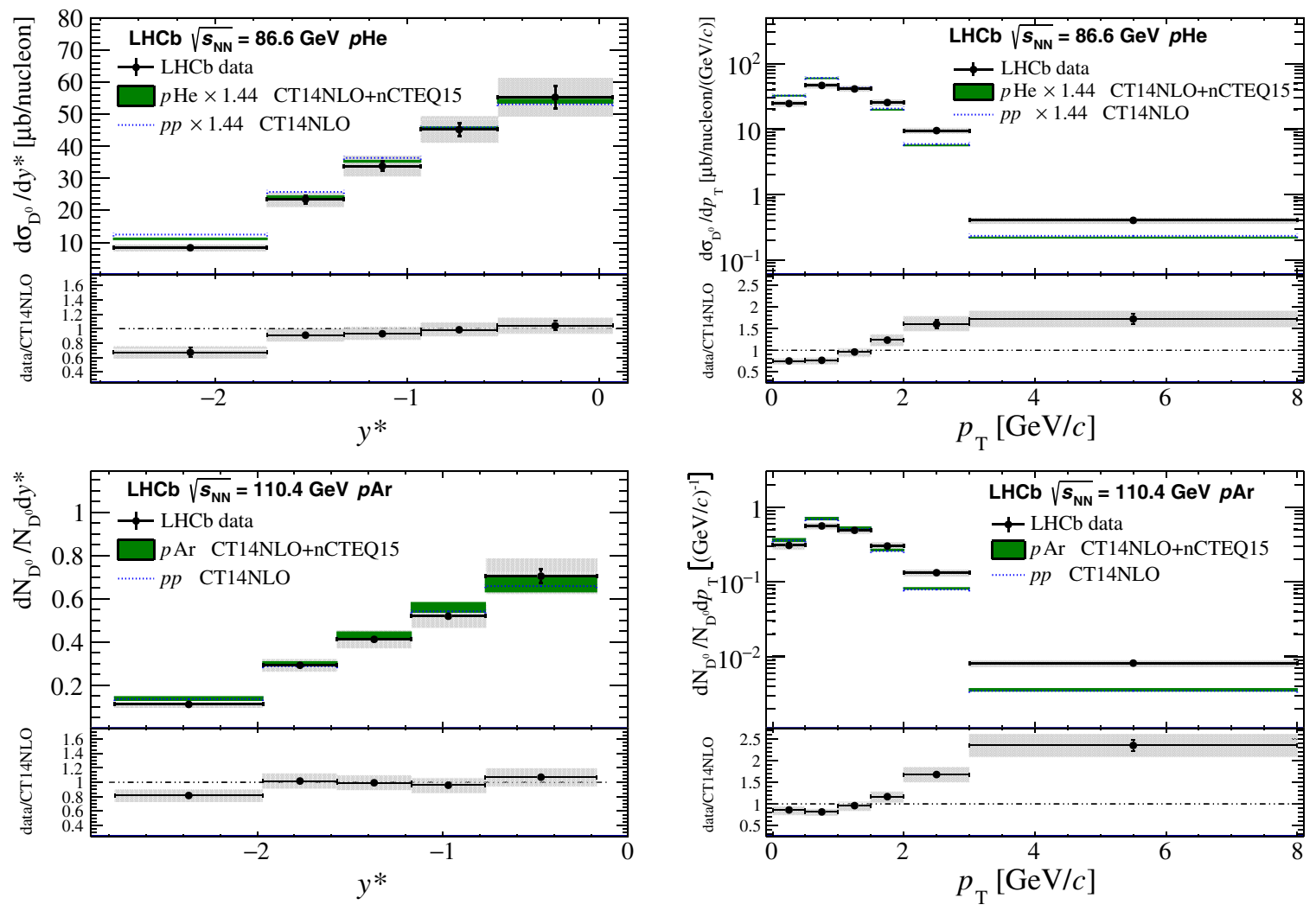

FIG. 4. Differential $D^{0}$ production cross sections for (top) $p \mathrm{He}$ and differential $D^{0}$ yields for (bottom) $p$ Ar collisions, as a function of (left) center-of-mass rapidity $y^{*}$ and (right) transverse momentum $p_{T}$. The data points mark the bin centers. The quadratic sum of statistical and uncorrelated systematic uncertainties are indicated by the vertical black lines. The correlated systematic uncertainties are indicated by the gray area. Theoretical predictions are described in the text. The lower panel of each plot shows the ratio of data to HELAC-ONIA $p p$ predictions.

Data are also compared with phenomenological parametrizations, interpolated to the present data energies, based on Refs. [12,50]. Solid and dashed red lines are obtained with linear and logarithmic interpolations, respectively, between the results from the $\mathrm{E} 789\left(p \mathrm{Au}, \sqrt{s_{\mathrm{NN}}}=38.7 \mathrm{GeV}\right)[51]$, HERA-B $\left(p \mathrm{C}, \sqrt{s_{\mathrm{NN}}}=41.5 \mathrm{GeV}\right)$ [52], and PHENIX ( $p p$, $\sqrt{s}=200 \mathrm{GeV}$ ) [53] experiments. The differential yields of $J / \psi$ as functions of $y^{*}$ and $p_{T}$, obtained from $p$ Ar data, are also shown in Fig. 3. Since the luminosity measurement is not available, only differential distributions with arbitrary normalization are shown.

The $D^{0}$ differential cross sections per target nucleon obtained for the $p \mathrm{He}$ dataset, as functions of $y^{*}$ and $p_{T}$, are shown in Fig. 4 and given in Ref. [36]. The HELAC-ONIA predictions underestimate the measured total cross section. The HELAC-ONIA predictions are rescaled by a factor 1.44 in Fig. 4 to compare the shape of the distributions. Differential yields, with arbitrary normalization, of $D^{0}$ as functions of $y^{*}$ and $p_{T}$ obtained from $p$ Ar data are also shown.

In fixed-target configuration, the $\mathrm{LHCb}$ acceptance gives access to the large Bjorken- $x$ region of the target nucleon (up to $x \sim 0.37$ for $D^{0}$ mesons). In this region, because of the small number of nucleons in the helium nucleus, nuclear effects affecting $c \bar{c}$ pairs are expected to be small. On the other hand, as suggested in Refs. [18,19], the intrinsic charm contribution, based on a valencelike parton distribution, can be substantial at large Bjorken- $x$. Using the approximation for $x$, the fraction of the nucleon momentum carried by the target parton,

$$
x \simeq \frac{2 m_{c}}{\sqrt{s_{\mathrm{NN}}}} \exp \left(-y^{*}\right)
$$

where $m_{c}=1.28 \mathrm{GeV} / c^{2}$ is the mass of the $c$ quark [54], the Bjorken- $x$ range $x \in[0.17,0.37]$ is obtained for the most backward bin. In this range any substantial intrinsic charm contribution should be seen in the $p \mathrm{He}$ results. As shown in Figs. 3 and 4, no strong differences are observed between $p$ He data and the theoretical predictions which do not include any intrinsic charm contribution. Therefore, within uncertainties, no evidence of substantial intrinsic charm content of the nucleon is observed in the data. Future measurements with larger samples and more accurate theoretical predictions will permit us to perform more quantitative studies, including the double-differential $\left[y^{*}, p_{\mathrm{T}}\right]$ production cross section.

In summary, we report the first measurement of heavy flavor production in fixed-target configuration at the LHC. 
The $J / \psi$ and $D^{0}$ production cross sections, measured in $p \mathrm{He}$ collisions at $\sqrt{s_{\mathrm{NN}}}=86.6 \mathrm{GeV}$ in the rapidity range $[2,4.6]$, are found to be $\sigma_{J / \psi}=652 \pm 33$ (stat) \pm 42 (syst) nb/ nucleon and $\sigma_{D^{0}}=80.8 \pm 2.4$ (stat) \pm 6.3 (syst) $\mu \mathrm{b} /$ nucleon. No evidence for a substantial intrinsic charm content of the nucleon is found.

We express our gratitude to our colleagues in the CERN accelerator departments for the excellent performance of the LHC. We thank the technical and administrative staff at the LHCb institutes. We acknowledge support from CERN and from the national agencies: CAPES, CNPq, FAPERJ and FINEP (Brazil); MOST and NSFC (China); CNRS/ IN2P3 (France); BMBF, DFG and MPG (Germany); INFN (Italy); NWO (Netherlands); MNiSW and NCN (Poland); MEN/IFA (Romania); MSHE (Russia); MinECo (Spain); SNSF and SER (Switzerland); NASU (Ukraine); STFC (United Kingdom); NSF (USA). We acknowledge the computing resources that are provided by CERN, IN2P3 (France), KIT and DESY (Germany), INFN (Italy), SURF (Netherlands), PIC (Spain), GridPP (United Kingdom), RRCKI and Yandex LLC (Russia), CSCS (Switzerland), IFIN-HH (Romania), CBPF (Brazil), PL-GRID (Poland) and OSC (USA). We are indebted to the communities behind the multiple open-source software packages on which we depend. Individual groups or members have received support from AvH Foundation (Germany); EPLANET, Marie Skłodowska-Curie Actions and ERC (European Union); ANR, Labex P2IO and OCEVU, and Région Auvergne-Rhône-Alpes (France); Key Research Program of Frontier Sciences of CAS, CAS PIFI, and the Thousand Talents Program (China); RFBR, RSF and Yandex LLC (Russia); GVA, XuntaGal and GENCAT (Spain); the Royal Society and the Leverhulme Trust (United Kingdom); Laboratory Directed Research and Development program of LANL (USA).

[1] A. Bazavov, Chiral crossover in QCD at zero and non-zero chemical potentials, arXiv:1812.08235.

[2] S. Digal, O. Kaczmarek, F. Karsch, and H. Satz, Heavy quark interactions in finite temperature QCD, Eur. Phys. J. C 43, 71 (2005).

[3] L. Kluberg and H. Satz, Color deconfinement and charmonium production in nuclear collisions, arXiv:0901.3831.

[4] H. Satz and K. Sridhar, Charmonium production versus open charm in nuclear collisions, Phys. Rev. D 50, 3557 (1994).

[5] Z. Conesa del Valle et al., Quarkonium production in high energy proton-proton and proton-nucleus collisions, Nucl. Phys. B, Proc. Suppl. 214, 3 (2011).

[6] E. G. Ferreiro, F. Fleuret, J. P. Lansberg, and A. Rakotozafindrabe, Cold nuclear matter effects on $J / \psi$ production: Intrinsic and extrinsic transverse momentum effects, Phys. Lett. B 680, 50 (2009).
[7] C. Lourenço, R. Vogt, and H. Wöhri, Energy dependence of $J / \psi$ absorption in proton-nucleus collisions, J. High Energy Phys. 02 (2009) 014.

[8] D. de Florian and R. Sassot, Nuclear parton distributions at next to leading order, Phys. Rev. D 69, 074028 (2004).

[9] K. J. Eskola, H. Paukkunen, and C. A. Salgado, EPS09: A new generation of NLO and LO nuclear parton distribution functions, J. High Energy Phys. 04 (2009) 065.

[10] J. L. Albacete and C. Marquet, Gluon saturation and initial conditions for relativistic heavy ion collisions, Prog. Part. Nucl. Phys. 76, 1 (2014).

[11] F. Arleo and S. Peigné, $J / \psi$ Suppression in $p-A$ Collisions from Parton Energy Loss in Cold QCD Matter, Phys. Rev. Lett. 109, 122301 (2012).

[12] F. Arleo, R. Kolevatov, S. Peigné, and M. Rustamova, Centrality and $p_{\perp}$ dependence of $J / \psi$ suppression in proton-nucleus collisions from parton energy loss, J. High Energy Phys. 05 (2013) 155.

[13] F. Arleo, S. Peigné, and T. Sami, Revisiting scaling properties of medium-induced gluon radiation, Phys. Rev. D 83, 114036 (2011).

[14] A. A. Alves, Jr. et al. (LHCb Collaboration), The LHCb detector at the LHC, J. Instrum. 3, S08005 (2008).

[15] R. Aaij et al. (LHCb Collaboration), LHCb detector performance, Int. J. Mod. Phys. A 30, 1530022 (2015).

[16] R. Aaij et al. (LHCb Collaboration), Precision luminosity measurements at LHCb, J. Instrum. 9, P12005 (2014).

[17] S. J. Brodsky, F. Fleuret, C. Hadjidakis, and J. P. Lansberg, Physics opportunities of a fixed-target experiment using LHC beams, Phys. Rep. 522, 239 (2013).

[18] J. Pumplin, H. L. Lai, and W. K. Tung, Charm parton content of the nucleon, Phys. Rev. D 75, 054029 (2007).

[19] S. Dulat, T.-J. Hou, J. Gao, J. Huston, J. Pumplin, C. Schmidt, D. Stump, and C.-P. Yuan, Intrinsic charm parton distribution functions from CTEQ-TEA global analysis, Phys. Rev. D 89, 073004 (2014).

[20] R. Aaij et al. (LHCb Collaboration), The LHCb trigger and its performance in 2011, J. Instrum. 8, P04022 (2013).

[21] R. Aaij et al. (LHCb Collaboration), Study of $J / \psi$ production and cold nuclear matter effects in $p P b$ collisions at $\sqrt{s_{\mathrm{NN}}}=5 \mathrm{TeV}$, J. High Energy Phys. 02 (2014) 072.

[22] LHCb Collaboration, Study of cold nuclear matter effects using prompt $D^{0}$ meson production in $p \mathrm{~Pb}$ collisions at LHCb, Report No. LHCb-CONF-2016-003, http://cds.cern .ch/record/2138946.

[23] T. Sjöstrand, S. Mrenna, and P. Skands, A brief introduction to Pythia 8.1, Comput. Phys. Commun. 178, 852 (2008).

[24] T. Sjöstrand, S. Mrenna, and P. Skands, PYTHIA 6.4 physics and manual, J. High Energy Phys. 05 (2006) 026.

[25] I. Belyaev et al. (LHCb Collaboration), Handling of the generation of primary events in Gauss, the LHCb simulation framework, J. Phys. Conf. Ser. 331, 032047 (2011).

[26] D. J. Lange, The EvtGen particle decay simulation package, Nucl. Instrum. Methods Phys. Res., Sect. A 462, 152 (2001).

[27] P. Golonka and Z. Was, PHOTOS Monte Carlo: A precision tool for QED corrections in $Z$ and $W$ decays, Eur. Phys. J. C 45, 97 (2006).

[28] T. Pierog, Iu. Karpenko, J. M. Katzy, E. Yatsenko, and K. Werner, EPOS LHC: Test of collective hadronization with 
data measured at the CERN Large Hadron Collider, Phys. Rev. C 92, 034906 (2015).

[29] J. Allison et al. (GEANT4 Collaboration), GEANT4 developments and applications, IEEE Trans. Nucl. Sci. 53, 270 (2006).

[30] S. Agostinelli et al. (GEAnt4 Collaboration), GEAnt4: A simulation toolkit, Nucl. Instrum. Methods Phys. Res. 506, 250 (2003).

[31] M. Clemencic, G. Corti, S. Easo, C. R. Jones, S. Miglioranzi, M. Pappagallo, and P. Robbe, The LHCb simulation application, Gauss: Design, evolution and experience, J. Phys. Conf. Ser. 331, 032023 (2011).

[32] R. Aaij et al. (LHCb Collaboration), Measurement of $J / \psi$ polarization in $p p$ collisions at $\sqrt{s}=7 \mathrm{TeV}$, Eur. Phys. J. C 73, 2631 (2013).

[33] A. Adare et al. (PHENIX Collaboration), Transverse momentum dependence of $J / \psi$ polarization at midrapidity in $p+p$ collisions at $\sqrt{s}=200 \mathrm{GeV}$, Phys. Rev. D 82, 012001 (2010).

[34] I. Abt et al. (HERA-B Collaboration), Angular distributions of leptons from $J / \psi$ 's produced in $920 \mathrm{GeV}$ fixed-target proton-nucleus collisions, Eur. Phys. J. C 60, 517 (2009).

[35] R. Aaij et al. (LHCb Collaboration), Measurement of forward $J / \psi$ production cross-sections in $p p$ collisions at $\sqrt{s}=13$ TeV, J. High Energy Phys. 10 (2015) 172; Erratum, J. High Energy Phys. 05 (2017) 63.

[36] See Supplemental Material at http://link.aps.org/ supplemental/10.1103/PhysRevLett.122.132002 for numerical values.

[37] T. Skwarnicki, A study of the radiative cascade transitions between the Upsilon-Prime and Upsilon resonances, Ph.D. thesis, Institute of Nuclear Physics, Krakow, 1986, DESYF31-86-02.

[38] L. Anderlini et al., The PIDCalib package, Report No. LHCbPUB-2016-021, http://cds.cern.ch/record/2202412.

[39] R. Aaij et al. (LHCb Collaboration), Measurement of Antiproton Production in $p \mathrm{He}$ Collisions at $\sqrt{s_{N N}}=$ 110 GeV, Phys. Rev. Lett. 121, 222001 (2018).

[40] H.-L. Lai, J. Huston, S. Mrenna, P. M. Nadolsky, D. Stump, W.-K. Tung, and C.-P. Yuan, Parton distributions for event generators, J. High Energy Phys. 04 (2010) 035.

[41] L. Gladilin, Fragmentation fractions of $c$ and $b$ quarks into charmed hadrons at LEP, Eur. Phys. J. C 75, 19 (2015).
[42] F. Maltoni et al., Analysis of charmonium production at fixed-target experiments in the NRQCD approach, Phys. Lett. B 638, 202 (2006).

[43] J. Adam et al. (ALICE Collaboration), $D$-meson production in $p$ - $\mathrm{Pb}$ collisions at $\sqrt{s_{N N}}=5.02 \mathrm{TeV}$ and in $p p$ collisions at $\sqrt{s}=7 \mathrm{TeV}$, Phys. Rev. C 94, 054908 (2016).

[44] M. L. Mangano, P. Nason, and G. Ridolfi, Heavy quark correlations in hadron collisions at next-to-leading order, Nucl. Phys. B373, 295 (1992).

[45] J.-P. Lansberg and H.-S. Shao, Towards an automated tool to evaluate the impact of the nuclear modification of the gluon density on quarkonium, D and B meson production in proton-nucleus collisions, Eur. Phys. J. C 77, 1 (2017).

[46] H.-S. Shao, HELAC-Onia 2.0: An upgraded matrix-element and event generator for heavy quarkonium physics, Comput. Phys. Commun. 198, 238 (2016).

[47] H.-S. Shao, HELAC-Onia: An automatic matrix element generator for heavy quarkonium physics, Comput. Phys. Commun. 184, 2562 (2013).

[48] S. Dulat, T.-J. Hou, J. Gao, M. Guzzi, J. Huston, P. Nadolsky, J. Pumplin, C. Schmidt, D. Stump, and C.-P. Yuan, New parton distribution functions from a global analysis of quantum chromodynamics, Phys. Rev. D 93, 033006 (2016).

[49] K. Kovarik et al., nCTEQ15: Global analysis of nuclear parton distributions with uncertainties in the CTEQ framework, Phys. Rev. D 93, 085037 (2016).

[50] F. Arleo and S. Peigné, Heavy-quarkonium suppression in p-A collisions from parton energy loss in cold QCD matter, J. High Energy Phys. 03 (2013) 122.

[51] M. H. Schub et al., Measurement of $J / \psi$ and $\psi^{\prime}$ production in $800 \mathrm{GeV}$ proton-gold collisions, Phys. Rev. D 52, 1307 (1995).

[52] I. Abt et al. (HERA-B Collaboration), Kinematic distributions and nuclear effects of $J / \psi$ production in $920 \mathrm{GeV}$ fixed-target proton-nucleus collisions, Eur. Phys. J. C 60, 525 (2009).

[53] A. Adare et al. (PHENIX Collaboration), $J / \psi$ Production Versus Transverse Momentum and Rapidity in $p+p$ Collisions at $\sqrt{s}=200 \mathrm{GeV}$, Phys. Rev. Lett. 98, 232002 (2007).

[54] M. Tanabashi et al. (Particle Data Group), 2018 Review of particle physics, Phys. Rev. D 98, 030001 (2018).

R. Aaij, ${ }^{27}$ B. Adeva, ${ }^{41}$ M. Adinolfi, ${ }^{48}$ C. A. Aidala, ${ }^{73}$ Z. Ajaltouni, ${ }^{5}$ S. Akar,${ }^{59}$ P. Albicocco, ${ }^{18}$ J. Albrecht,${ }^{10}$ F. Alessio, ${ }^{42}$ M. Alexander, ${ }^{53}$ A. Alfonso Albero, ${ }^{40} \mathrm{~S}$. Ali, ${ }^{27} \mathrm{G}$. Alkhazov, ${ }^{33}$ P. Alvarez Cartelle, ${ }^{55}$ A. A. Alves Jr., ${ }^{41}$ S. Amato, ${ }^{2}$ S. Amerio, ${ }^{23}$ Y. Amhis, ${ }^{7}$ L. An,${ }^{3}$ L. Anderlini, ${ }^{17}$ G. Andreassi, ${ }^{43}$ M. Andreotti, ${ }^{16, g}$ J. E. Andrews,${ }^{60}$ R. B. Appleby, ${ }^{56}$ F. Archilli, ${ }^{27}$ P. d'Argent, ${ }^{12}$ J. Arnau Romeu, ${ }^{6}$ A. Artamonov, ${ }^{39}$ M. Artuso, ${ }^{61}$ K. Arzymatov, ${ }^{37}$ E. Aslanides, ${ }^{6}$ M. Atzeni, ${ }^{44}$ B. Audurier, ${ }^{22}$ S. Bachmann, ${ }^{12}$ J. J. Back, ${ }^{50}$ S. Baker, ${ }^{55}$ V. Balagura, ${ }^{7, b}$ W. Baldini, ${ }^{16}$ A. Baranov, ${ }^{37}$ R. J. Barlow, ${ }^{56}$ S. Barsuk, ${ }^{7}$ W. Barter, ${ }^{56}$ F. Baryshnikov, ${ }^{70}$ V. Batozskaya, ${ }^{31}$ B. Batsukh, ${ }^{61}$ V. Battista ${ }^{43}$ A. Bay, ${ }^{43}$ J. Beddow,${ }^{53}$ F. Bedeschi, ${ }^{24}$ I. Bediaga,${ }^{1}$ A. Beiter,${ }^{61}$ L. J. Bel,${ }^{27}$ S. Belin, ${ }^{22}$ N. Beliy, ${ }^{63}$ V. Bellee ${ }^{43}$ N. Belloli, ${ }^{20, i}$ K. Belous,${ }^{39}$ I. Belyaev, ${ }^{34,42}$ E. Ben-Haim, ${ }^{8}$ G. Bencivenni, ${ }^{18}$ S. Benson, ${ }^{27}$ S. Beranek, ${ }^{9}$ A. Berezhnoy, ${ }^{35}$ R. Bernet, ${ }^{44}$ D. Berninghoff, ${ }^{12}$ E. Bertholet, ${ }^{8}$ A. Bertolin, ${ }^{23}$ C. Betancourt, ${ }^{44}$ F. Betti,${ }^{15,42}$ M. O. Bettler, ${ }^{49}$ M. van Beuzekom, ${ }^{27}$ Ia. Bezshyiko, ${ }^{44}$ S. Bhasin, ${ }^{48}$ 
J. Bhom, ${ }^{29}$ S. Bifani, ${ }^{47}$ P. Billoir, ${ }^{8}$ A. Birnkraut, ${ }^{10}$ A. Bizzeti,,${ }^{17, u}$ M. Bjørn,${ }^{57}$ M. P. Blago, ${ }^{42}$ T. Blake, ${ }^{50}$ F. Blanc, ${ }^{43}$ S. Blusk,${ }^{61}$ D. Bobulska, ${ }^{53}$ V. Bocci, ${ }^{26}$ O. Boente Garcia, ${ }^{41}$ T. Boettcher, ${ }^{58}$ A. Bondar, ${ }^{38, w}$ N. Bondar, ${ }^{33}$ S. Borghi, ${ }^{56,42}$ M. Borisyak, ${ }^{37}$ M. Borsato, ${ }^{41}$ F. Bossu, ${ }^{7}$ M. Boubdir, ${ }^{9}$ T. J. V. Bowcock, ${ }^{54}$ C. Bozzi,${ }^{16,42}$ S. Braun, ${ }^{12}$ M. Brodski, ${ }^{42}$ J. Brodzicka, ${ }^{29}$ A. Brossa Gonzalo, ${ }^{50}$ D. Brundu, ${ }^{22}$ E. Buchanan, ${ }^{48}$ A. Buonaura, ${ }^{44}$ C. Burr, ${ }^{56}$ A. Bursche,${ }^{22}$ J. Buytaert, ${ }^{42}$ W. Byczynski, ${ }^{42}$ S. Cadeddu, ${ }^{22}$ H. Cai,${ }^{64}$ R. Calabrese, ${ }^{16, \mathrm{~g}}$ R. Calladine, ${ }^{47}$ M. Calvi, ${ }^{20, \mathrm{i}}$ M. Calvo Gomez ${ }^{40, \mathrm{~m}}$ A. Camboni,${ }^{40, \mathrm{~m}}$ P. Campana, ${ }^{18}$ D. H. Campora Perez, ${ }^{42}$ L. Capriotti, ${ }^{56}$ A. Carbone,${ }^{15, e}$ G. Carboni, ${ }^{25}$ R. Cardinale, ${ }^{19, h}$ A. Cardini, ${ }^{22}$ P. Carniti, ${ }^{20, i}$ L. Carson, ${ }^{52}$ K. Carvalho Akiba, ${ }^{2}$ G. Casse, ${ }^{54}$ L. Cassina, ${ }^{20}$ M. Cattaneo, ${ }^{42}$ G. Cavallero, ${ }^{19, \mathrm{~h}}$ R. Cenci, ${ }^{24, \mathrm{p}}$ D. Chamont, ${ }^{7}$ M. G. Chapman, ${ }^{48}$ M. Charles,${ }^{8}$ Ph. Charpentier, ${ }^{42}$ G. Chatzikonstantinidis, ${ }^{47}$ M. Chefdeville, ${ }^{4}$ V. Chekalina,${ }^{37}$ C. Chen, ${ }^{3}$ S. Chen, ${ }^{22}$ S.-G. Chitic, ${ }^{42}$ V. Chobanova, ${ }^{41}$ M. Chrzaszcz,${ }^{42}$ A. Chubykin, ${ }^{33}$ P. Ciambrone, ${ }^{18}$ X. Cid Vidal, ${ }^{41}$ G. Ciezarek, ${ }^{42}$ P. E. L. Clarke, ${ }^{52}$ M. Clemencic, ${ }^{42}$ H. V. Cliff, ${ }^{49}$ J. Closier, ${ }^{42}$ V. Coco, ${ }^{42}$ J. A. B. Coelho, ${ }^{7}$ J. Cogan, ${ }^{6}$ E. Cogneras, ${ }^{5}$

L. Cojocariu, ${ }^{32}$ P. Collins, ${ }^{42}$ T. Colombo, ${ }^{42}$ A. Comerma-Montells, ${ }^{12}$ A. Contu, ${ }^{22}$ G. Coombs, ${ }^{42}$ S. Coquereau, ${ }^{40}$ G. Corti, ${ }^{42}$ M. Corvo, ${ }^{16, g}$ C. M. Costa Sobral,${ }^{50}$ B. Couturier, ${ }^{42}$ G. A. Cowan, ${ }^{52}$ D. C. Craik,${ }^{58}$ A. Crocombe,${ }^{50}$ M. Cruz Torres, ${ }^{1}$ R. Currie ${ }^{52}$ C. D’Ambrosio, ${ }^{42}$ F. Da Cunha Marinho, ${ }^{2}$ C. L. Da Silva, ${ }^{74}$ E. Dall'Occo, ${ }^{27}$ J. Dalseno, ${ }^{48}$ A. Danilina, ${ }^{34}$ A. Davis, ${ }^{3}$ O. De Aguiar Francisco, ${ }^{42}$ K. De Bruyn, ${ }^{42}$ S. De Capua,${ }^{56}$ M. De Cian, ${ }^{43}$ J. M. De Miranda, ${ }^{1}$ L. De Paula, ${ }^{2}$ M. De Serio, ${ }^{14, d}$ P. De Simone, ${ }^{18}$ C. T. Dean, ${ }^{53}$ D. Decamp, ${ }^{4}$ L. Del Buono, ${ }^{8}$ B. Delaney, ${ }^{49}$ H.-P. Dembinski, ${ }^{11}$ M. Demmer, ${ }^{10}$ A. Dendek, ${ }^{30}$ D. Derkach,${ }^{37}$ O. Deschamps,${ }^{5}$ F. Desse, ${ }^{7}$ F. Dettori, ${ }^{54}$ B. Dey,${ }^{65}$ A. Di Canto, ${ }^{42}$ P. Di Nezza,${ }^{18}$ S. Didenko, ${ }^{70}$ H. Dijkstra, ${ }^{42}$ F. Dordei ${ }^{42}$ M. Dorigo, ${ }^{42, x}$ A. Dosil Suárez, ${ }^{41}$ L. Douglas, ${ }^{53}$ A. Dovbnya, ${ }^{45}$ K. Dreimanis,${ }^{54}$ L. Dufour ${ }^{27}$ G. Dujany, ${ }^{8}$ P. Durante, ${ }^{42}$ J. M. Durham, ${ }^{74}$ D. Dutta ${ }^{56}$ R. Dzhelyadin, ${ }^{39}$ M. Dziewiecki, ${ }^{12}$ A. Dziurda,${ }^{29}$ A. Dzyuba, ${ }^{33}$ S. Easo, ${ }^{51}$ U. Egede ${ }^{55}$ V. Egorychev, ${ }^{34}$ S. Eidelman, ${ }^{38, w}$ S. Eisenhardt, ${ }^{52}$ U. Eitschberger, ${ }^{10}$ R. Ekelhof, ${ }^{10}$ L. Eklund, ${ }^{53}$ S. Ely, ${ }^{61}$ A. Ene, ${ }^{32}$ S. Escher, ${ }^{9}$ S. Esen, ${ }^{27}$ T. Evans, ${ }^{59}$ A. Falabella, ${ }^{15}$ N. Farley ${ }^{47}$ S. Farry, ${ }^{54}$ D. Fazzini, ${ }^{20,42, i}$ L. Federici, ${ }^{25}$ P. Fernandez Declara, ${ }^{42}$ A. Fernandez Prieto, ${ }^{41}$ F. Ferrari, ${ }^{15}$ L. Ferreira Lopes, ${ }^{43}$ F. Ferreira Rodrigues, ${ }^{2}$ M. Ferro-Luzzi, ${ }^{42}$ S. Filippov,${ }^{36}$ R. A. Fini, ${ }^{14}$ M. Fiorini, ${ }^{16, g}$ M. Firlej ${ }^{30}$ C. Fitzpatrick,${ }^{43}$ T. Fiutowski, ${ }^{30}$ F. Fleuret,,${ }^{7, b}$ M. Fontana, ${ }^{22,42}$ F. Fontanelli, ${ }^{19, \mathrm{~h}}$ R. Forty, ${ }^{42}$ V. Franco Lima, ${ }^{54}$ M. Frank, ${ }^{42}$ C. Frei, ${ }^{42}$ J. Fu, ${ }^{21, q}$ W. Funk, ${ }^{42}$ C. Färber, ${ }^{42}$ M. Féo,${ }^{27}$ E. Gabriel, ${ }^{52}$

A. Gallas Torreira, ${ }^{41}$ D. Galli, ${ }^{15, \mathrm{e}} \mathrm{S}$. Gallorini, ${ }^{23} \mathrm{~S}$. Gambetta, ${ }^{52}$ Y. Gan, ${ }^{3}$ M. Gandelman, ${ }^{2}$ P. Gandini, ${ }^{21}$ Y. Gao, ${ }^{3}$ L. M. Garcia Martin, ${ }^{72}$ B. Garcia Plana, ${ }^{41}$ J. García Pardiñas, ${ }^{44}$ J. Garra Tico, ${ }^{49}$ L. Garrido, ${ }^{40}$ D. Gascon ${ }^{40}$ C. Gaspar, ${ }^{42}$ L. Gavardi, ${ }^{10}$ G. Gazzoni, ${ }^{5}$ D. Gerick, ${ }^{12}$ E. Gersabeck, ${ }^{56}$ M. Gersabeck, ${ }^{56}$ T. Gershon, ${ }^{50}$ D. Gerstel,,${ }^{6}$ Ph. Ghez, ${ }^{4}$ S. Gianì, ${ }^{43}$ V. Gibson, ${ }^{49}$ O. G. Girard, ${ }^{43}$ L. Giubega ${ }^{32}$ K. Gizdov, ${ }^{52}$ V. V. Gligorov, ${ }^{8}$ D. Golubkov, ${ }^{34}$ A. Golutvin, ${ }^{55,70}$ A. Gomes, ${ }^{1, a}$ I. V. Gorelov, ${ }^{35}$ C. Gotti, ${ }^{20, i}$ E. Govorkova, ${ }^{27}$ J. P. Grabowski, ${ }^{12}$ R. Graciani Diaz,${ }^{40}$ L. A. Granado Cardoso, ${ }^{42}$ E. Graugés,${ }^{40}$ E. Graverini, ${ }^{44}$ G. Graziani, ${ }^{17}$ A. Grecu, ${ }^{32}$ R. Greim, ${ }^{27}$ P. Griffith, ${ }^{22}$ L. Grillo, ${ }^{56}$ L. Gruber, ${ }^{42}$ B. R. Gruberg Cazon, ${ }^{57}$ O. Grünberg, ${ }^{67}$ C. Gu, ${ }^{3}$ E. Gushchin, ${ }^{36}$ Yu. Guz,${ }^{39,42}$ T. Gys,${ }^{42}$ C. Göbel, ${ }^{62}$ T. Hadavizadeh ${ }^{57}$ C. Hadjivasiliou, ${ }^{5}$ G. Haefeli, ${ }^{43}$

C. Haen, ${ }^{42}$ S. C. Haines, ${ }^{49}$ B. Hamilton, ${ }^{60}$ X. Han ${ }^{12}$ T. H. Hancock, ${ }^{57}$ S. Hansmann-Menzemer, ${ }^{12}$ N. Harnew, ${ }^{57}$

S. T. Harnew, ${ }^{48}$ T. Harrison, ${ }^{54}$ C. Hasse,${ }^{42}$ M. Hatch, ${ }^{42}$ J. He, ${ }^{63}$ M. Hecker, ${ }^{55}$ K. Heinicke, ${ }^{10}$ A. Heister, ${ }^{10}$ K. Hennessy,${ }^{54}$ L. Henry, ${ }^{72}$ E. van Herwijnen, ${ }^{42}$ M. Heß,${ }^{67}$ A. Hicheur, ${ }^{2}$ R. Hidalgo Charman, ${ }^{56}$ D. Hill, ${ }^{57}$ M. Hilton, ${ }^{56}$ P. H. Hopchev, ${ }^{43}$ W. Hu ${ }^{65}$ W. Huang ${ }^{63}$ Z. C. Huard, ${ }^{59}$ W. Hulsbergen, ${ }^{27}$ T. Humair, ${ }^{55}$ M. Hushchyn, ${ }^{37}$ D. Hutcheroft, ${ }^{54}$ D. Hynds, ${ }^{27}$ P. Ibis, ${ }^{10}$ M. Idzik, ${ }^{30}$ P. Ilten, ${ }^{47}$ K. Ivshin, ${ }^{33}$ R. Jacobsson, ${ }^{42}$ J. Jalocha, ${ }^{57}$ E. Jans, ${ }^{27}$ A. Jawahery, ${ }^{60}$ F. Jiang, ${ }^{3}$ M. John, ${ }^{57}$ D. Johnson, ${ }^{42}$ C. R. Jones, ${ }^{49}$ C. Joram, ${ }^{42}$ B. Jost, ${ }^{42}$ N. Jurik,${ }^{57}$ S. Kandybei, ${ }^{45}$ M. Karacson, ${ }^{42}$ J. M. Kariuki, ${ }^{48}$ S. Karodia, ${ }^{53}$ N. Kazeev,${ }^{37}$ M. Kecke, ${ }^{12}$ F. Keizer, ${ }^{49}$ M. Kelsey, ${ }^{61}$ M. Kenzie, ${ }^{49}$ T. Ketel, ${ }^{28}$ E. Khairullin, ${ }^{37}$ B. Khanji, ${ }^{12}$ C. Khurewathanakul, ${ }^{43}$

K. E. Kim, ${ }^{61}$ T. Kirn, ${ }^{9}$ S. Klaver, ${ }^{18}$ K. Klimaszewski, ${ }^{31}$ T. Klimkovich, ${ }^{11}$ S. Koliiev, ${ }^{46}$ M. Kolpin, ${ }^{12}$ R. Kopecna, ${ }^{12}$ P. Koppenburg, ${ }^{27}$ I. Kostiuk, ${ }^{27}$ S. Kotriakhova, ${ }^{33}$ M. Kozeiha, ${ }^{5}$ L. Kravchuk, ${ }^{36}$ M. Kreps, ${ }^{50}$ F. Kress, ${ }^{55}$ P. Krokovny, ${ }^{38, w}$ W. Krupa, ${ }^{30}$ W. Krzemien, ${ }^{31}$ W. Kucewicz, ${ }^{29,1}$ M. Kucharczyk, ${ }^{29}$ V. Kudryavtsev, ${ }^{38, w}$ A. K. Kuonen, ${ }^{43}$ T. Kvaratskheliya,${ }^{34,42}$ D. Lacarrere, ${ }^{42}$ G. Lafferty, ${ }^{56}$ A. Lai, ${ }^{22}$ D. Lancierini, ${ }^{44}$ G. Lanfranchi, ${ }^{18}$ C. Langenbruch, ${ }^{9}$ T. Latham, ${ }^{50}$ C. Lazzeroni, ${ }^{47}$ R. Le Gac, ${ }^{6}$ A. Leflat, ${ }^{35}$ J. Lefrançois, ${ }^{7}$ R. Lefèvre, ${ }^{5}$ F. Lemaitre, ${ }^{42}$ O. Leroy, ${ }^{6}$ T. Lesiak, ${ }^{29}$ B. Leverington, ${ }^{12}$ P.-R. Li, ${ }^{63}$ T. Li,${ }^{3}$ Z. Li ${ }^{61}$ X. Liang, ${ }^{61}$ T. Likhomanenko, ${ }^{69}$ R. Lindner, ${ }^{42}$ F. Lionetto, ${ }^{44}$ V. Lisovskyi, ${ }^{7}$ X. Liu, ${ }^{3}$ D. Loh, ${ }^{50}$ A. Loi, ${ }^{22}$ I. Longstaff, ${ }^{53}$ J. H. Lopes, ${ }^{2}$ G. H. Lovell, ${ }^{49}$ D. Lucchesi, ${ }^{23,0}$ M. Lucio Martinez, ${ }^{41}$ A. Lupato, ${ }^{23}$ E. Luppi, ${ }^{16, g}$ O. Lupton, ${ }^{42}$ A. Lusiani, ${ }^{24}$ X. Lyu ${ }^{63}$ F. Machefert, ${ }^{7}$ F. Maciuc,${ }^{32}$ V. Macko, ${ }^{43}$ P. Mackowiak, ${ }^{10}$ S. Maddrell-Mander ${ }^{48}$ O. Maev, ${ }^{33,42}$ K. Maguire, ${ }^{56}$ D. Maisuzenko, ${ }^{33}$ M. W. Majewski, ${ }^{30}$ S. Malde, ${ }^{57}$ B. Malecki, ${ }^{29}$ A. Malinin, ${ }^{69}$ T. Maltsev, ${ }^{38, w}$ G. Manca, ${ }^{22, f}$ G. Mancinelli, ${ }^{6}$ D. Marangotto, ${ }^{21, q}$ J. Maratas, ${ }^{5, v}$ J. F. Marchand, ${ }^{4}$ U. Marconi, ${ }^{15}$ C. Marin Benito, ${ }^{7}$ M. Marinangeli, ${ }^{43}$ 
P. Marino, ${ }^{43}$ J. Marks, ${ }^{12}$ P. J. Marshall,${ }^{54}$ G. Martellotti, ${ }^{26}$ M. Martin,${ }^{6}$ M. Martinelli, ${ }^{42}$ D. Martinez Santos,${ }^{41}$ F. Martinez Vidal, ${ }^{72}$ A. Massafferri, ${ }^{1}$ M. Materok, ${ }^{9}$ R. Matev, ${ }^{42}$ A. Mathad,${ }^{50}$ Z. Mathe, ${ }^{42}$ C. Matteuzzi, ${ }^{20}$ A. Mauri, ${ }^{44}$ E. Maurice, ${ }^{7, b}$ B. Maurin, ${ }^{43}$ A. Mazurov, ${ }^{47}$ M. McCann, ${ }^{55,42}$ A. McNab,${ }^{56}$ R. McNulty, ${ }^{13}$ J. V. Mead,${ }^{54}$ B. Meadows, ${ }^{59}$ C. Meaux ${ }^{6}$ F. Meier,${ }^{10}$ N. Meinert, ${ }^{67}$ D. Melnychuk, ${ }^{31}$ M. Merk, ${ }^{27}$ A. Merli, ${ }^{21, q}$ E. Michielin, ${ }^{23}$ D. A. Milanes, ${ }^{66}$ E. Millard, ${ }^{50}$ M.-N. Minard, ${ }^{4}$ L. Minzoni ${ }^{16, g}$ D. S. Mitzel, ${ }^{12}$ A. Mogini ${ }^{8}$ J. Molina Rodriguez, ${ }^{1, y}$ T. Mombächer, ${ }^{10}$ I. A. Monroy, ${ }^{66}$ S. Monteil,${ }^{5}$ M. Morandin, ${ }^{23}$ G. Morello, ${ }^{18}$ M. J. Morello, ${ }^{24, t}$ O. Morgunova, ${ }^{69}$ J. Moron, ${ }^{30}$ A. B. Morris, ${ }^{6}$ R. Mountain, ${ }^{61}$ F. Muheim, ${ }^{52}$ M. Mulder,${ }^{27}$ C. H. Murphy, ${ }^{57}$ D. Murray, ${ }^{56}$ A. Mödden, ${ }^{10}$ D. Müller, ${ }^{42}$ J. Müller, ${ }^{10}$ K. Müller, ${ }^{44}$ V. Müller, ${ }^{10}$ P. Naik, ${ }^{48}$ T. Nakada, ${ }^{43}$ R. Nandakumar, ${ }^{51}$ A. Nandi, ${ }^{57}$ T. Nanut ${ }^{43}$ I. Nasteva, ${ }^{2}$ M. Needham, ${ }^{52}$ N. Neri, ${ }^{21}$ S. Neubert, ${ }^{12}$ N. Neufeld, ${ }^{42}$ M. Neuner, ${ }^{12}$ T. D. Nguyen, ${ }^{43}$ C. Nguyen-Mau, ${ }^{43, n}$ S. Nieswand, ${ }^{9}$ R. Niet,${ }^{10}$ N. Nikitin, ${ }^{35}$ A. Nogay ${ }^{69}$ N. S. Nolte, ${ }^{42}$ D. P. O'Hanlon, ${ }^{15}$ A. Oblakowska-Mucha, ${ }^{30}$ V. Obraztsov, ${ }^{39}$ S. Ogilvy, ${ }^{18}$ R. Oldeman, ${ }^{22, f}$ C. J. G. Onderwater, ${ }^{68}$ A. Ossowska, ${ }^{29}$ J. M. Otalora Goicochea, ${ }^{2}$ P. Owen, ${ }^{44}$ A. Oyanguren, ${ }^{72}$ P. R. Pais, ${ }^{43}$ T. Pajero, ${ }^{24, t}$ A. Palano, ${ }^{14}$ M. Palutan, ${ }^{18,42}$ G. Panshin, ${ }^{71}$ A. Papanestis, ${ }^{51}$ M. Pappagallo, ${ }^{52}$ L. L. Pappalardo, ${ }^{16, g}$ W. Parker,${ }^{60}$ C. Parkes,${ }^{56}$ G. Passaleva, ${ }^{17,42}$ A. Pastore,${ }^{14}$ M. Patel,${ }^{55}$ C. Patrignani,${ }^{15, e}$ A. Pearce,${ }^{42}$ A. Pellegrino, ${ }^{27}$ G. Penso, ${ }^{26}$ M. Pepe Altarelli, ${ }^{42}$ S. Perazzini, ${ }^{42}$ D. Pereima, ${ }^{34}$ P. Perret, ${ }^{5}$ L. Pescatore,${ }^{43}$ K. Petridis, ${ }^{48}$ A. Petrolini, ${ }^{19, h}$ A. Petrov, ${ }^{69}$ S. Petrucci, ${ }^{52}$ M. Petruzzo, ${ }^{21, q}$ B. Pietrzyk, ${ }^{4}$ G. Pietrzyk, ${ }^{43}$ M. Pikies, ${ }^{29}$ M. Pili, ${ }^{57}$ D. Pinci, ${ }^{26}$ J. Pinzino, ${ }^{42}$ F. Pisani, ${ }^{42}$ A. Piucci, ${ }^{12}$ V. Placinta, ${ }^{32}$ S. Playfer, ${ }^{52}$ J. Plews, ${ }^{47}$ M. Plo Casasus, ${ }^{41}$ F. Polci, ${ }^{8}$ M. Poli Lener, ${ }^{18}$ A. Poluektov,${ }^{50}$ N. Polukhina ${ }^{70, c}$ I. Polyakov, ${ }^{61}$ E. Polycarpo, ${ }^{2}$ G. J. Pomery, ${ }^{48}$ S. Ponce, ${ }^{42}$ A. Popov, ${ }^{39}$ D. Popov,${ }^{47,11}$ S. Poslavskii, ${ }^{39}$ C. Potterat, ${ }^{2}$ E. Price, ${ }^{48}$ J. Prisciandaro, ${ }^{41}$ C. Prouve, ${ }^{48}$ V. Pugatch, ${ }^{46}$ A. Puig Navarro, ${ }^{44}$ H. Pullen, ${ }^{57}$ G. Punzi,${ }^{24, p}$ W. Qian, ${ }^{63}$ J. Qin, ${ }^{63}$ R. Quagliani, ${ }^{8}$ B. Quintana, ${ }^{5}$ B. Rachwal, ${ }^{30}$ J. H. Rademacker, ${ }^{48}$ M. Rama, ${ }^{24}$ M. Ramos Pernas, ${ }^{41}$ M. S. Rangel, ${ }^{2}$ F. Ratnikov, ${ }^{37, a b}$ G. Raven, ${ }^{28}$ M. Ravonel Salzgeber, ${ }^{42}$ M. Reboud, ${ }^{4}$ F. Redi, ${ }^{43}$ S. Reichert, ${ }^{10}$ A. C. dos Reis, ${ }^{1}$ F. Reiss, ${ }^{8}$ C. Remon Alepuz, ${ }^{72}$ Z. Ren, ${ }^{3}$ V. Renaudin, ${ }^{7}$ S. Ricciardi, ${ }^{51}$ S. Richards, ${ }^{48}$ K. Rinnert, ${ }^{54}$ P. Robbe, ${ }^{7}$ A. Robert ${ }^{8}$ A. B. Rodrigues, ${ }^{43}$ E. Rodrigues,${ }^{59}$ J. A. Rodriguez Lopez ${ }^{66}$ M. Roehrken, ${ }^{42}$ A. Rogozhnikov, ${ }^{37}$ S. Roiser, ${ }^{42}$ A. Rollings, ${ }^{57}$ V. Romanovskiy, ${ }^{39}$ A. Romero Vidal, ${ }^{41}$ M. Rotondo, ${ }^{18}$ M. S. Rudolph ${ }^{61}$ T. Ruf, ${ }^{42}$ J. Ruiz Vidal, ${ }^{72}$ J. J. Saborido Silva, ${ }^{41}$ N. Sagidova, ${ }^{33}$ B. Saitta, ${ }^{22, f}$ V. Salustino Guimaraes, ${ }^{62}$ C. Sanchez Gras, ${ }^{27}$ C. Sanchez Mayordomo, ${ }^{72}$ B. Sanmartin Sedes,${ }^{41}$ R. Santacesaria, ${ }^{26}$ C. Santamarina Rios, ${ }^{41}$ M. Santimaria, ${ }^{18}$ E. Santovetti,${ }^{25, j}$ G. Sarpis,${ }^{56}$ A. Sarti, ${ }^{18, k}$ C. Satriano, ${ }^{26, s}$ A. Satta, ${ }^{25}$ M. Saur, ${ }^{63}$ D. Savrina, ${ }^{34,35}$ S. Schael, ${ }^{9}$ M. Schellenberg, ${ }^{10}$ M. Schiller, ${ }^{53}$ H. Schindler, ${ }^{42}$ M. Schmelling, ${ }^{11}$ T. Schmelzer, ${ }^{10}$ B. Schmidt, ${ }^{42}$ O. Schneider, ${ }^{43}$ A. Schopper, ${ }^{42}$ H. F. Schreiner, ${ }^{59}$ M. Schubiger, ${ }^{43}$ M. H. Schune, ${ }^{7}$ R. Schwemmer, ${ }^{42}$ B. Sciascia, ${ }^{18}$ A. Sciubba, ${ }^{26, k}$ A. Semennikov, ${ }^{34}$ E. S. Sepulveda, ${ }^{8}$ A. Sergi, ${ }^{47,42}$ N. Serra, ${ }^{44}$ J. Serrano, ${ }^{6}$ L. Sestini, ${ }^{23}$ A. Seuthe, ${ }^{10}$ P. Seyfert, ${ }^{42}$ M. Shapkin, ${ }^{39}$ Y. Shcheglov, ${ }^{33, \dagger}$ T. Shears, ${ }^{54}$ L. Shekhtman, ${ }^{38, w}$ V. Shevchenko, ${ }^{69}$ E. Shmanin, ${ }^{70}$ B. G. Siddi, ${ }^{16}$ R. Silva Coutinho, ${ }^{44}$ L. Silva de Oliveira, ${ }^{2}$ G. Simi,${ }^{23, o}$ S. Simone,${ }^{14, d}$ N. Skidmore, ${ }^{12}$ T. Skwarnicki, ${ }^{61}$ J. G. Smeaton, ${ }^{49}$ E. Smith, ${ }^{9}$ I. T. Smith, ${ }^{52}$ M. Smith,${ }^{55}$ M. Soares, ${ }^{15}$ 1. Soares Lavra, ${ }^{1}$ M. D. Sokoloff, ${ }^{59}$ F. J. P. Soler, ${ }^{53}$ B. Souza De Paula, ${ }^{2}$ B. Spaan, ${ }^{10}$ P. Spradlin, ${ }^{53}$ F. Stagni, ${ }^{42}$ M. Stahl,${ }^{12}$ S. Stahl,${ }^{42}$ P. Stefko, ${ }^{43}$ S. Stefkova, ${ }^{55}$ O. Steinkamp, ${ }^{44}$ S. Stemmle, ${ }^{12}$ O. Stenyakin, ${ }^{39}$ M. Stepanova, ${ }^{33}$ H. Stevens, ${ }^{10}$ A. Stocchi, ${ }^{7}$ S. Stone,${ }^{61}$ B. Storaci, ${ }^{44}$ S. Stracka, ${ }^{24, p}$ M. E. Stramaglia, ${ }^{43}$ M. Straticiuc, ${ }^{32}$ U. Straumann, ${ }^{44}$ S. Strokov, ${ }^{71}$ J. Sun, ${ }^{3}$ L. Sun, ${ }^{64}$ K. Swientek, ${ }^{30}$ V. Syropoulos, ${ }^{28}$ T. Szumlak, ${ }^{30}$ M. Szymanski, ${ }^{63}$ S. T'Jampens, ${ }^{4}$ Z. Tang, ${ }^{3}$ A. Tayduganov, ${ }^{6}$ T. Tekampe, ${ }^{10}$ G. Tellarini,${ }^{16}$ F. Teubert, ${ }^{42}$ E. Thomas, ${ }^{42}$ J. van Tilburg, ${ }^{27}$ M. J. Tilley, ${ }^{55}$ V. Tisserand, ${ }^{5}$ M. Tobin,${ }^{30}$ S. Tolk, ${ }^{42}$ L. Tomassetti, ${ }^{16, g}$ D. Tonelli, ${ }^{24}$ D. Y. Tou, ${ }^{8}$ R. Tourinho Jadallah Aoude, ${ }^{1}$ E. Tournefier, ${ }^{4}$ M. Traill, ${ }^{53}$ M. T. Tran, ${ }^{43}$ A. Trisovic, ${ }^{49}$ A. Tsaregorodtsev, ${ }^{6}$ G. Tuci, ${ }^{24}$ A. Tully, ${ }^{49}$ N. Tuning, ${ }^{27,42}$ A. Ukleja, ${ }^{31}$ A. Usachov, ${ }^{7}$ A. Ustyuzhanin, ${ }^{37}$ U. Uwer, ${ }^{12}$ A. Vagner, ${ }^{71}$ V. Vagnoni, ${ }^{15}$ A. Valassi ${ }^{42}$ S. Valat,${ }^{42}$ G. Valenti, ${ }^{15}$ R. Vazquez Gomez, ${ }^{42}$ P. Vazquez Regueiro, ${ }^{41}$ S. Vecchi, ${ }^{16}$ M. van Veghel, ${ }^{27}$ J. J. Velthuis, ${ }^{48}$ M. Veltri, ${ }^{17, \mathrm{r}}$ G. Veneziano, ${ }^{57}$ A. Venkateswaran, ${ }^{61}$ T. A. Verlage, ${ }^{9}$ M. Vernet, ${ }^{5}$ M. Veronesi, ${ }^{27}$ N. V. Veronika, ${ }^{13}$ M. Vesterinen, ${ }^{57}$ J. V. Viana Barbosa, ${ }^{42}$ D. Vieira, ${ }^{63}$ M. Vieites Diaz, ${ }^{41}$ H. Viemann, ${ }^{67}$ X. Vilasis-Cardona, ${ }^{40, m}$ A. Vitkovskiy, ${ }^{27}$ M. Vitti, ${ }^{49}$ V. Volkov, ${ }^{35}$ A. Vollhardt, ${ }^{44}$ B. Voneki, ${ }^{42}$ A. Vorobyev, ${ }^{33}$ V. Vorobyev, ${ }^{38, w}$ J. A. de Vries,${ }^{27}$ C. Vázquez Sierra, ${ }^{27}$ R. Waldi, ${ }^{67}$ J. Walsh,${ }^{24}$ J. Wang, ${ }^{61}$ M. Wang, ${ }^{3}$ Y. Wang, ${ }^{65}$ Z. Wang,${ }^{44}$ D. R. Ward ${ }^{49}$ H. M. Wark, ${ }^{54}$ N. K. Watson, ${ }^{47}$ D. Websdale, ${ }^{55}$ A. Weiden, ${ }^{44}$ C. Weisser, ${ }^{58}$ M. Whitehead, ${ }^{9}$ J. Wicht ${ }^{50}$ G. Wilkinson, ${ }^{57}$ M. Wilkinson, ${ }^{61}$ I. Williams, ${ }^{49}$ M. R. J. Williams, ${ }^{56}$ M. Williams, ${ }^{58}$ T. Williams, ${ }^{47}$ F. F. Wilson, ${ }^{51,42}$ J. Wimberley, ${ }^{60}$ M. Winn, ${ }^{7}$ J. Wishahi, ${ }^{10}$ W. Wislicki,${ }^{31}$ M. Witek, ${ }^{29}$ G. Wormser, ${ }^{7}$ S. A. Wotton, ${ }^{49}$ K. Wyllie, ${ }^{42}$ D. Xiao ${ }^{65}$ Y. Xie, ${ }^{65}$ A. Xu, ${ }^{3}$ M. Xu, ${ }^{65}$ Q. Xu, ${ }^{63}$ Z. Xu, ${ }^{3}$ Z. Xu, ${ }^{4}$ Z. Yang, ${ }^{3}$ Z. Yang, ${ }^{60}$ Y. Yao, ${ }^{61}$ L. E. Yeomans,${ }^{54}$ H. Yin, ${ }^{65}$ J. Yu, ${ }^{65, \text { aa }}$ 
X. Yuan, ${ }^{61}$ O. Yushchenko, ${ }^{39}$ K. A. Zarebski, ${ }^{47}$ M. Zavertyaev, ${ }^{11, c}$ D. Zhang, ${ }^{65}$ L. Zhang, ${ }^{3}$ W. C. Zhang, ${ }^{3, z}$ Y. Zhang, ${ }^{7}$ A. Zhelezov, ${ }^{12}$ Y. Zheng, ${ }^{63}$ X. Zhu, ${ }^{3}$ V. Zhukov, ${ }^{9,35}$ J. B. Zonneveld, ${ }^{52}$ and S. Zucchelli ${ }^{15}$

(LHCb Collaboration)

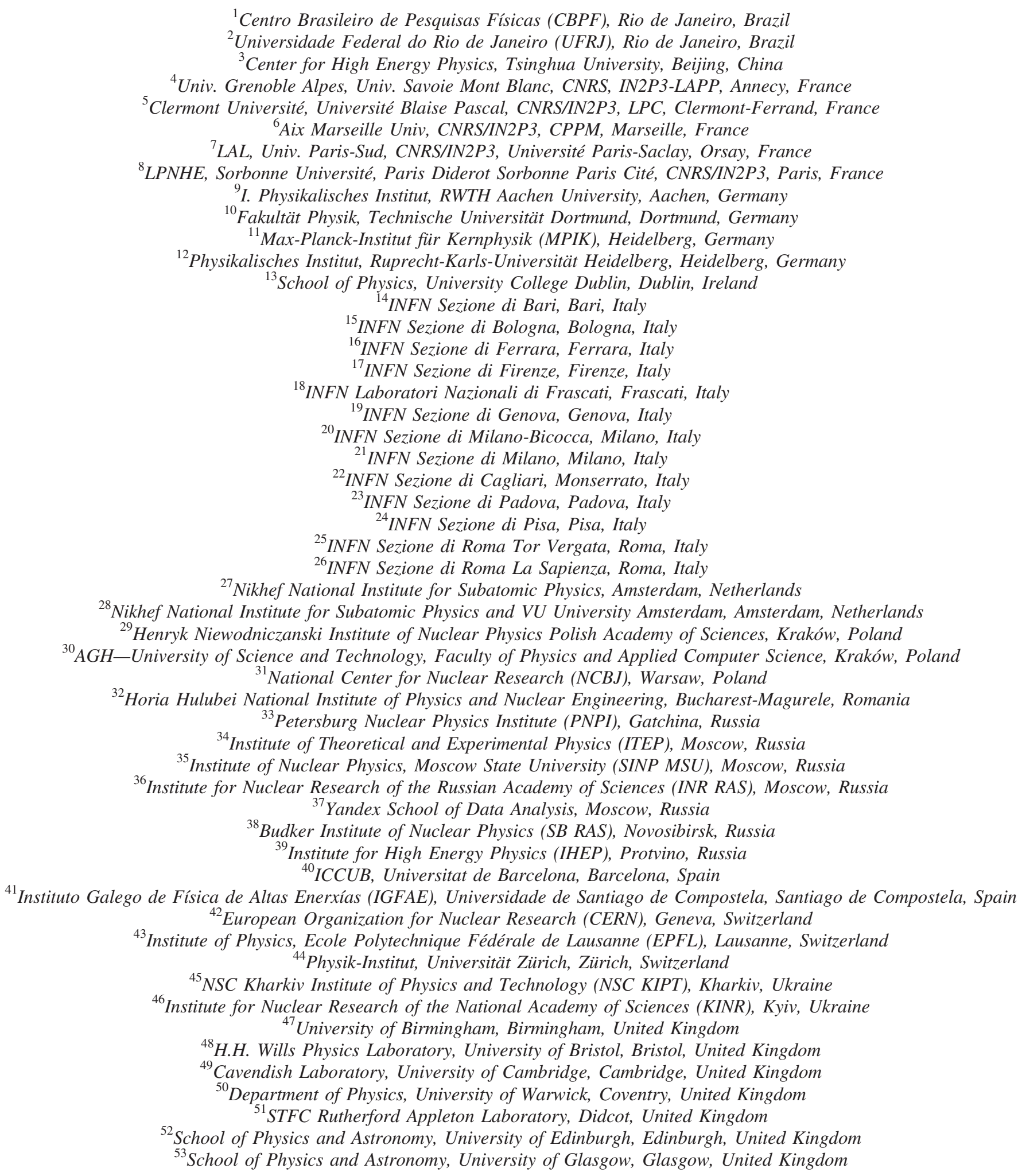




\author{
${ }^{54}$ Oliver Lodge Laboratory, University of Liverpool, Liverpool, United Kingdom \\ ${ }^{55}$ Imperial College London, London, United Kingdom \\ ${ }^{56}$ School of Physics and Astronomy, University of Manchester, Manchester, United Kingdom \\ ${ }^{57}$ Department of Physics, University of Oxford, Oxford, United Kingdom \\ ${ }^{58}$ Massachusetts Institute of Technology, Cambridge, Massachusetts, USA \\ ${ }^{59}$ University of Cincinnati, Cincinnati, Ohio, USA \\ ${ }^{60}$ University of Maryland, College Park, Maryland, USA \\ ${ }^{61}$ Syracuse University, Syracuse, New York, USA \\ ${ }^{62}$ Pontifícia Universidade Católica do Rio de Janeiro (PUC-Rio), Rio de Janeiro, Brazil \\ [associated with Universidade Federal do Rio de Janeiro (UFRJ), Rio de Janeiro, Brazil] \\ ${ }^{63}$ University of Chinese Academy of Sciences, Beijing, China \\ (associated with Center for High Energy Physics, Tsinghua University, Beijing, China) \\ ${ }^{64}$ School of Physics and Technology, Wuhan University, Wuhan, China \\ (associated with Center for High Energy Physics, Tsinghua University, Beijing, China) \\ ${ }^{65}$ Institute of Particle Physics, Central China Normal University, Wuhan, Hubei, China \\ (associated with Center for High Energy Physics, Tsinghua University, Beijing, China) \\ ${ }^{66}$ Departamento de Fisica, Universidad Nacional de Colombia, Bogota, Colombia \\ (associated with LPNHE, Sorbonne Université, Paris Diderot Sorbonne Paris Cité, CNRS/IN2P3, Paris, France) \\ ${ }^{67}$ Institut für Physik, Universität Rostock, Rostock, Germany \\ (associated with Physikalisches Institut, Ruprecht-Karls-Universität Heidelberg, Heidelberg, Germany) \\ ${ }^{68}$ Van Swinderen Institute, University of Groningen, Groningen, Netherlands \\ (associated with Nikhef National Institute for Subatomic Physics, Amsterdam, Netherlands) \\ ${ }^{69}$ National Research Centre Kurchatov Institute, Moscow, Russia \\ [associated with Institute of Theoretical and Experimental Physics (ITEP), Moscow, Russia] \\ ${ }^{70}$ National University of Science and Technology "MISIS", Moscow, Russia \\ [associated with Institute of Theoretical and Experimental Physics (ITEP), Moscow, Russia] \\ ${ }^{71}$ National Research Tomsk Polytechnic University, Tomsk, Russia \\ [associated with Institute of Theoretical and Experimental Physics (ITEP), Moscow, Russia] \\ ${ }^{72}$ Instituto de Fisica Corpuscular, Centro Mixto Universidad de Valencia-CSIC, Valencia, Spain \\ (associated with ICCUB, Universitat de Barcelona, Barcelona, Spain) \\ ${ }^{73}$ University of Michigan, Ann Arbor, USA \\ (associated with Syracuse University, Syracuse, New York, USA) \\ ${ }^{74}$ Los Alamos National Laboratory (LANL), Los Alamos, USA \\ (associated with Syracuse University, Syracuse, New York, USA)
}

${ }^{\dagger}$ Deceased.

${ }^{a}$ Also at Universidade Federal do Triângulo Mineiro (UFTM), Uberaba-MG, Brazil.

${ }^{\mathrm{b}}$ Also at Laboratoire Leprince-Ringuet, Palaiseau, France.

${ }^{\mathrm{c}}$ Also at P.N. Lebedev Physical Institute, Russian Academy of Science (LPI RAS), Moscow, Russia.

${ }^{\mathrm{d}}$ Also at Università di Bari, Bari, Italy.

${ }^{\mathrm{e}}$ Also at Università di Bologna, Bologna, Italy.

${ }^{\mathrm{f}}$ Also at Università di Cagliari, Cagliari, Italy.

${ }^{\mathrm{g}}$ Also at Università di Ferrara, Ferrara, Italy.

${ }^{\mathrm{h}}$ Also at Università di Genova, Genova, Italy.

${ }^{\mathrm{i}}$ Also at Università di Milano Bicocca, Milano, Italy.

${ }^{\mathrm{j}}$ Also at Università di Roma Tor Vergata, Roma, Italy.

${ }^{\mathrm{k}}$ Also at Università di Roma La Sapienza, Roma, Italy.

${ }^{1}$ Also at AGH-University of Science and Technology, Faculty of Computer Science, Electronics and Telecommunications, Kraków, Poland.

${ }^{\mathrm{m}}$ Also at LIFAELS, La Salle, Universitat Ramon Llull, Barcelona, Spain.

${ }^{\mathrm{n}}$ Also at Hanoi University of Science, Hanoi, Vietnam.

${ }^{\circ}$ Also at Università di Padova, Padova, Italy.

${ }^{\mathrm{P}}$ Also at Università di Pisa, Pisa, Italy.

${ }^{\mathrm{q}}$ Also at Università degli Studi di Milano, Milano, Italy.

${ }^{\mathrm{r}}$ Also at Università di Urbino, Urbino, Italy.



${ }^{\mathrm{t}}$ Also at Scuola Normale Superiore, Pisa, Italy.

${ }^{\text {u}}$ Also at Università di Modena e Reggio Emilia, Modena, Italy.

${ }^{v}$ Also at MSU-Iligan Institute of Technology (MSU-IIT), Iligan, Philippines.

${ }^{\mathrm{w}}$ Also at Novosibirsk State University, Novosibirsk, Russia. 


\footnotetext{
${ }^{\mathrm{x}}$ Also at Sezione INFN di Trieste, Trieste, Italy.

${ }^{y}$ Also at Escuela Agrícola Panamericana, San Antonio de Oriente, Honduras.

${ }^{\mathrm{z}}$ Also at School of Physics and Information Technology, Shaanxi Normal University (SNNU), Xi'an, China.

${ }^{\text {aa }}$ Also at Physics and Micro Electronic College, Hunan University, Changsha City, China.

${ }^{\mathrm{ab}}$ Also at National Research University Higher School of Economics, Moscow, Russia.
} 